\title{
Materialien über die Ökologie und Biologie von Sphaeromides Bureschi Strouhal
}

\author{
Von Angel Angelovi)
}

Mit Tafel 28 (1)

Die letzten zwei Jahrzehnte trugen viel zum Kennenlernen der bulgarischen Höhlenfauna bei. Es wurden neue Gattungen und Arten von Höhlenorganismen entdeckt, darunter auch Vertreter der Wasserhöhlenfauna. Zusammen mit einigen mikroskopischen und kleinen Wasserorganismen wurde auch der für unsere Höhlen seiner Größe nach an dritter Stelle stehende (nach Pelodrilus bureschi und Dina lineata arndti) Höhlenwasserorganismus, die Isopode aus der Gattung Sphaeromides, entdeckt.

Nach Material, das von Akademiker Ivan Buresch in den Höhlen der West-Staraplanina bei den Dörfern Iskrez und Zerovo gesammelt wurde, beschrieb Strouhal einen neuen Vertreter der Gattung Sphaeromides, den er zu Ehren seines Entdeckers Sphaeromides bureschi benannte. In seiner Arbeit beschäftigt sich Strouhal (1963) fast ausschließlich mit den morphologischen Merkmalen dieses außergewöhnlich interessanten Organismus. Bezüglich dessen Ökologie und Biologie erwähnt er fast nichts, mit Ausnahme des Biotopentypus, in welchem die beschriebenen Exemplare aufgefunden wurden, und zwar einen unterirdischen Fluß.

Die ergänzend gesammelten Angaben bezüglich der Ökologie und Biologie dieser Isopode, desgleichen auch das Auffinden einer dritten Fundstätte derselben, ergaben das Material zu vorliegender Abhandlung.

Die bisherigen zwei Fundstätten von Sphaeromides bureschi waren an erster Stelle der See und der unterirdische Fluß in der Höhle ,,Duschnik" beim Dorfe Iskrez, weiterhin der unterirdische Fluß in der „Wasser-Höhle“ bei der Bahnhaltestelle Zerovo.

Beim Studium der Fauna zweier benachbarter Gruppen großer Karstquellen, bei den Dörfern Opizvet und Besden (25 km westlich von Sofia) wurde in den Jahren 1959 und 1964 festgestellt, daß das Wasser der ersten Quellengruppe (beim Dorfe Opizvet) außer einigen

\footnotetext{
1) Zoologisches Institut der Universität, ul. Moskovska 49 - Sofia (Bulgarien).
} 
anderen Troglobionten auch noch Sphaeromides bureschi beherbergt. So wurde im Monat September 1959 mit einem Netz, das an den Trichter einer der Quellen zum Fang von Troglobionten angebracht wurde, ein ô Exemplar aufgefangen und im Jahre 1964 konnten einige Male auf dieselbe Weise noch 6 Exemplare - alles 우 - gesammelt werden.

Alle drei Fundstätten befinden sich im Rayon der West-Staraplanina; die ersten zwei in Trias-, die dritte in Malmkalkstein. Obwohl bis jetzt in der Nähe der dritten, neuen Fundstätte keine Höhleneingänge entdeckt werden konnten, muß man analog zu den ersten zwei annehmen, daß auch hier Sphaeromides bureschi große unterirdische Seen und Flüsse besiedelt und dies um so mehr, da nach nicht publizierten Angaben des Hydroingenieurs P. Petrov sich im Schoß des Karstgebietes oberhalb der Dörfer Opizvet und Besden große Wasserbecken befinden. Aber die Tatsache, daß die unterirdischen Gewässer verhältnismäßig viele Exemplare dieser Isopode mit sich nach außen führen, spricht mehr für eine unmittelbare Nähe von Quellen eines unterirdischen Flusses als für das Vorhandensein eines unterirdischen Sees.

Es ist interessant, daß die Trichter der Quellenreihe bei dem Dorfe Opizvet jenen aus dem Dorfe Besden benachbart sind und auf einund derselben Kote liegen; einige davon befinden sich in unmittelbarer Nähe zueinander. Die Gewässer dieser zwei Quellengruppen geben, wenigstens ihren Merkmalen nach, keinen Grund zur Annahme, daß sie unterirdisch im Zusammenhang stehen. Als Resultat von gleichzeitig entnommenen Proben im Verlaufe eines Jahres (1964) wurde in den Gewässern aus den Quellen bei Opizvet zusammen mit Sphaeromides bureschi auch Plagigeyeria gladilini procerulus festgestellt. Das Quellwasser beim Dorfe Besden führte kein einziges Exemplar von den oben erwähnten Arten mit sich, aber statt dessen einige Exemplare von der Art Stenasellus sp. und Bureschia sp. Gemeinsam für beide Quellengruppen erwies sich nur eine Art Niphargus sp.

Um die Ökologie und Biologie von Sphaeromides bureschi eingehender zu beleuchten, wurden einige Besuche der, ,Wasser-Höhle" bei der Bahnhaltestelle Zerovo unternommen, und zwar an den folgenden Tagen: 16. XII. 1963; 8. III., 18. VI. und 9. VIII. 1964. Gleichzeitig damit wurden auch einige Beobachtungen unter Laboratoriumsbedingungen in Aquarien durchgeführt, die in Dunkelheit und bei niedriger Temperatur belassen wurden.

Bei den wiederholten Besuchen der Höhle von Zerovo (,WasserHöhle") wurde von mir und von anderen Erforschern dieser Höhle festgestellt, daß Sphaeromides bureschi, 50-100 m vom Eingang ent- 
fernt, im ganzen Lauf des Höhlenflusses, der über $2000 \mathrm{~m}$ lang ist, angetroffen wird. Hier besiedelt diese Isopode hauptsächlich diejenigen Plätze, die aus sandigem Grund bestehen und an denen die Strömung nicht stärker als $0,50 \mathrm{~m} / \mathrm{sec}$ ist (an der Wasseroberfläche gemessen). Es sind dies gewöhnlich Plätze, an denen der Fluß sich verbreitert oder an Tiefe zunimmt, so daß in der Grundwasserschicht kaum eine Strömung zu verspüren ist.

Diese Tendenz von Sphaeromides bureschi, stille und sandige Teile des Höhlenflusses aufzusuchen, wird besonders dann ersichtlich, wenn ein Vergleich mit den Plätzen vorgenommen wird, die von der zweiten Isopode bewohnt werden, und zwar von Bureschia bulgarica, die sich im gleichen Fluß aufhält. Letztere besiedelt Biotope mit kieseligen und kieselig-sandigem Grund, dort wo die Stromschnelligkeit 0,75 bis $1,00 \mathrm{~m} / \mathrm{sec}$ beträgt und auch am Grund fühlbar zu verspüren ist.

In ihren Biotopen wird Sphaeromides bureschi in Gruppen von 3-5 Exemplaren beobachtet. Im allgemeinen ist die Menge der Individuen in der Höhle groß. So wurde bei Besuchen, die an verschiedenen Tagen vorgenommen wurden, festgestellt, daß sich die Menge dieses Isopoden auf ungefähr 1-10 Stück pro 100 m Flußlänge beläuft. Auch die Höhle „,Duschnik“ weist einen verhältnismäßig großen Reichtum an Exemplaren auf, das heißt im ersten See, bei einer Fläche von ungefähr $50 \mathrm{~m}^{2}$, wurden über 20 Exemplare aufgefunden.

Eine Unterscheidung der beiden Geschlechter bei Sphaeromides bureschi kann leicht an ihrem Standort schon vorgenommen werden, und dies nicht nur auf Grund der zwischen ihnen bestehenden Größen-

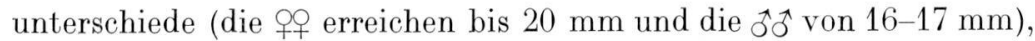
sondern auch auf Grund inner-anatomischer Merkmale, die leicht durch den Panzer der Tiere, der durchsichtig wie Glas ist, zu beobachten sind. So scheinen im hinteren Körperteil der männlichen Individuen die milchweißen gewundenen Samenleiter (vas deferens) sehr klar hindurch. Bei durchgeführten Beobachtungen über das Verhältnis zwischen männlichen zu den weiblichen Individuen wurde ermittelt, daß letztere an Zahl fast immer die männlichen überragen. So wurden zum Beispiel am 8. VIII. 1964 unter 17 gesammelten Exemplaren 16 weibliche und nur ein männliches festgestellt.

Das verhältnismäßig häufige Vorkommen dieser Höhlenassel an den beiden Fundstätten, besonders in der, ,Wasser-Höhle" bei Zerovo, ist wahrscheinlich auf den Überfluß an Nahrung zurückzuführen, die von außen in die Höhle gelangt, und zwar durch Erdoberflächenwasser, das in die Höhle eindringt und durch reichlich klebenbleibendes Guano. Durch Proben, die vom Höhlenfluß zwecks Untersuchung der 
Organismenwelt entnommen wurden, konnte die reichliche Anwesenheit von Larven aus der Fam. Tendipedidae, desgleichen auch von Larven von Ephemeroptera und Oligochaeta festgestellt werden.

Außer diesen Organismen, die nicht nur einmalig im Magen von sezierten Sphaeromiden festgestellt wurden, konnten auch weiche Chitinteile von ausgewachsenen Insekten beobachtet werden, die vermittels des Guano in das Wasser gerieten.

Bei den von der, ,Wasser-Höhle“ gebotenen Bedingungen bei Zerovo lebt Sphaeromides bureschi bei einer Wassertemperatur in den Grenzen von $9,6^{\circ}-13,5^{\circ} \mathrm{C}$, mit einem Sauerstoffgehalt von $5,5-6 \mathrm{~cm}^{3}$ pro Liter Wasser, einer aktiven Reaktion $(\mathrm{pH})$ von 6,5-7 und Härte des Wassers von $26,6^{\circ}$ in deutschen Graden.

Die unterirdischen Gewässer bei einigen der Quellen im Dorfe Opizvet weisen höhere Wassertemperatur auf und erreichen bis zu $21^{\circ}$ C. Aber dort, wo Sphaeromides bureschi gesammelt wurde, zeigt die Wassertemperatur $15-16^{\circ} \mathrm{C}, \mathrm{pH}=7$ und allgemeine Härte $22^{\circ} \mathrm{H}$.

Aus letzterwähnten Angaben ist zu ersehen, daß Sphaeromides bureschi normal bei einer ziemlich hohen Wassertemperatur leben kann. Bei nicht natürlichen Bedingungen - beim Befördern dieser Assel in der Thermosflasche - hält sie eine Temperatur von $18-19^{\circ}$ aus. Bei Aquariumsbedingungen bewegt sie sich aktiv bei einer Temperatur von $16,2^{\circ} \mathrm{C}$ und nimmt gerne Nahrung zu sich. Niedrige Temperaturen bei Aquariumsbedingungen stören ihre normale Lebensweise. Bei Temperaturen unter $8^{\circ} \mathrm{C}$ werden sie schwer beweglich und bei $4,5-5^{\circ} \mathrm{C}$ erstarren sie und reagieren kaum auf eine Reizung.

Sphaeromides bureschi, obwohl auf den ersten Blick ziemlich plump und schwer beweglich, kann bei Störung sehr aktiv eine Strecke von 20-30 cm schwimmen. Das Schwimmen geht hauptsächlich mit Hilfe der Pleopoden vor sich. Dieselben stoßen beim schnellen Andrücken an den Bauch das sich zwischen ihnen befindliche Wasser heraus, wobei der Körper einen Sprung nach vorne macht.

Ungeachtet einer sorgfältigen Prüfung aller gesammelten Exemplare auf Ektoparasiten oder Kommensale wurden bis jetzt auf den Rändern der Pleopoden nur auf einigen Individuen ein paar Exemplare von Acineta sp. festgestellt. Obwohl Sphaeromides bureschi bei Aquariumsbedingungen verhältnismäßig leicht und ohne besondere Mühe länger als ein Jahr gehalten werden kann, und sich in den ihr gegebenen weiten Temperaturgrenzen wohl befindet, ihre Nahrung, Enchytraeidae oder Stückchen anderen frischen Fleisches mit Appetit verzehrt, verbleibt ihre Fortpflanzungsweise bis jetzt unseren Beobachtungen entzogen. 


\section{LITERATUR}

Strounal, H. - 1963: Sphaeromides bureschi, eine neue Höhlen-Wasserassel aus Bulgarien (Isopoda, Cirolanidae). Bulg. Akad. Nauk., Izvest. zool. Inst. Muz. Sofia, 13, 157-175.

\section{ZUSAMMENFASSUNG}

Die Höhlen-Wasserassel Sphaeromides bureschi Strouhal ist von I. Buresch in den unterirdischen Gewässern zweier Höhlen der West-Staraplanina entdeckt worden. Der Verfasser beschreibt hier einen neuen Biotop: es handelt sich um eine in diesem Gebiet gelegene Quelle. Die Ergebnisse der Beobachtungen, die ersten über die Ökologie und Biologie dieser Isopoden (Biotop, Temperatur, Zahlenverhältnis der Geschlechter, Nahrungsbedingungen, Bewegungsart) werden mitgeteilt.

\section{RÉSUMÉ}

L'Isopode aquatique cavernicole Sphaeromides bureschi Strouhal fut découvert par I. Buresch dans les eaux souterraines de deux grottes de la Stara-Planina occidentale. Dans cette note, l'auteur décrit une nouvelle station constituée par une source située dans la même région, puis expose le résultat de ses observations, les premières qui aient été faites sur l'écologie et la biologie de cet Isopode (biotope, température, sex-ratio, régime alimentaire, locomotion).

\section{ERKLÄRUNG DER TAFEL 28 (1):}

Sphaeromides bureschi Strouhal: unten ein Paar; oben ein Männchen. 

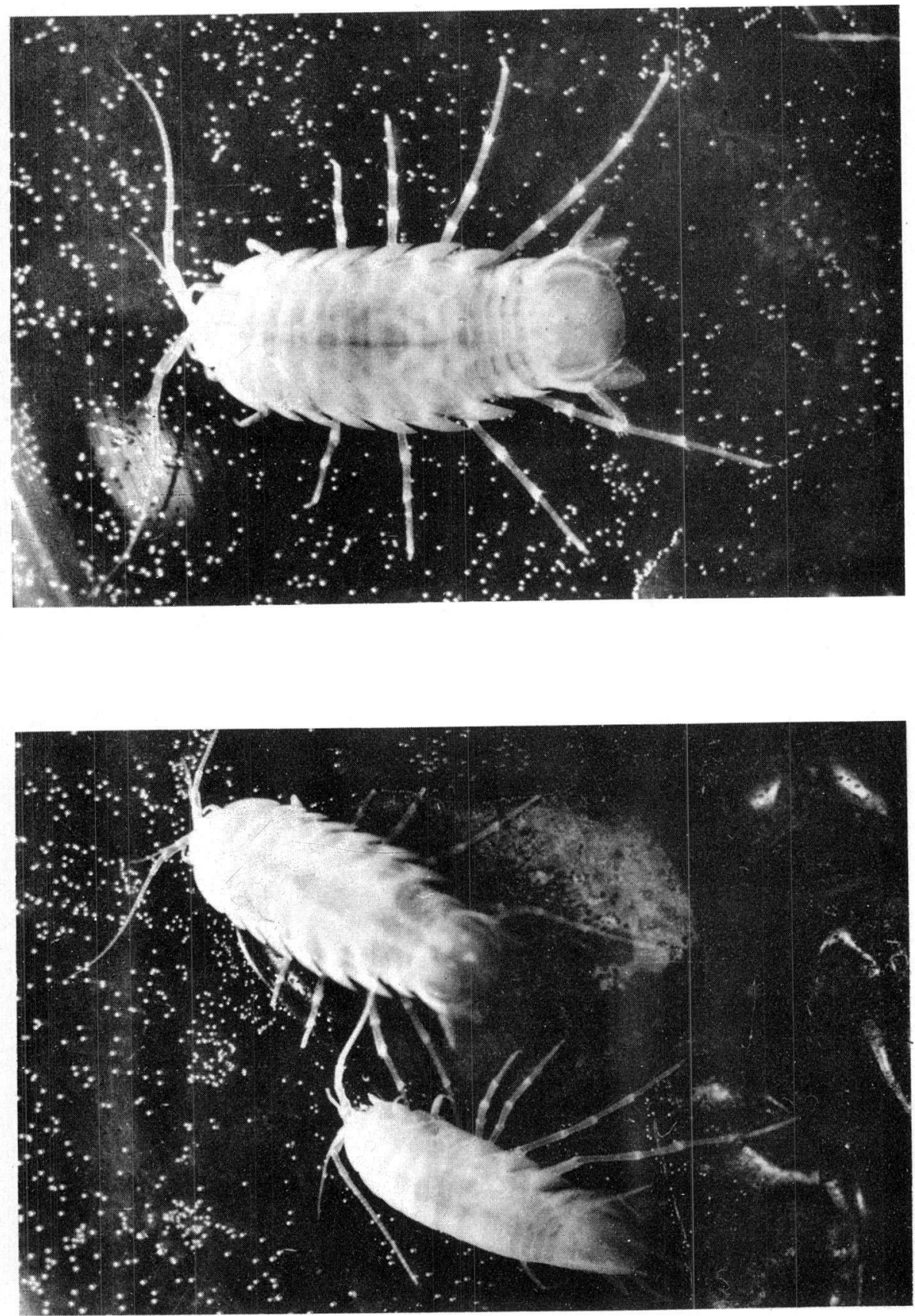